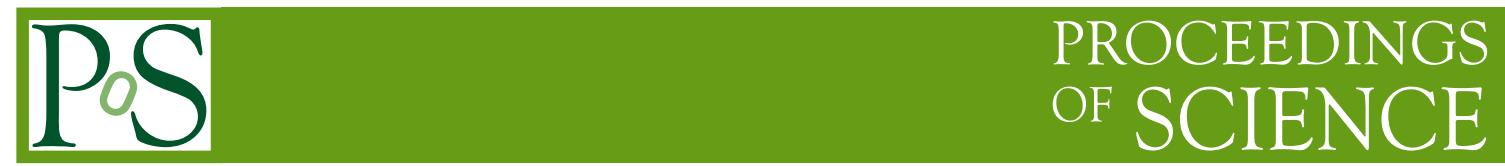

\title{
POWHEG: status and perspectives
}

\author{
Simone ALIOLI*广 \\ DESY \\ E-mail: Simone.alioliedesy.de
}

I review recent developments and applications of the POWHEG method, to merge NLO calculations with Shower Monte Carlo programs, in the POWHEG-BOX framework. Particular emphasis is given to the recent implementation of the top-quark pairs hadroproduction in association with a hard jet.

The 2011 Europhysics Conference on High Energy Physics-HEP 2011,

July 21-27, 2011

Grenoble, Rhône-Alpes France

\footnotetext{
* Speaker.

${ }^{\dagger}$ Based on works done in collaboration with K. Hamilton, S. Moch, P. Nason, C. Oleari, E. Re and P. Uwer
} 


\section{The POWHEG method and the POWHEG BOX}

In present-days particle physics phenomenology it is important to provide accurate and fully exclusive theoretical predictions for the direct comparison with experimental data. Due to the inherent limitations of the fixed-order approaches, that are unable to provide reliable hadron-level predictions for exclusive observables, in the past shower Monte Carlo (SMC) programs - mostly with leading-order accuracy only - were used for this task. Nowadays, much better theoretical precision can be reached by merging next-to-leading (NLO) computations with parton showers. A merging method, that avoids the double counting of emissions, has been pioneered by MC@NLO [1] and later on followed by POWHEG [2,3]. The two approaches combine the accuracy of exact hard matrix elements for the large angle scattering - including all the radiative corrections to first order in the strong coupling constant $\alpha_{s}$ - with the soft and collinear emission described by the parton shower. In this way, they achieve both a reduced sensitivity with respect to variations of the unphysical renormalization and factorization scales, the correct Sudakov suppression of collinear and soft emissions and, basing on phenomenological models of hadrons formation, they produce realistic events with the same final state particles that can be observed in data. At variance with MC@NLO, the POWHEG method allows for the generation of positive weighted events only and is also independent of the parton SMC generator used. This independence is achieved by generating the hardest radiation, i.e. that with the highest transverse momentum, first and then letting the SMC perform the remaining shower, but requiring subsequent emissions not to be harder than the first.

An automatic tool, dubbed POWHEG BOX [4], is available to ease the POWHEG implementation of new processes. It only requires as input the individual components of the NLO calculation under consideration, i.e., the Born process, its virtual radiative corrections and the real emission contributions. Then it automatically combines them, canceling the emerging soft and collinear singularities in the Frixione-Kunszt-Signer (FKS) subtraction scheme, and produce the required events. The POWHEG BOX may also be seen as a library, where previously implemented processes are available in a common framework. Recent addictions include jet pair production [5], $W^{+} W^{+}$ plus dijet production [6,7], diboson [8] and $W b \bar{b}$ [9] production. For an up-to-date list of included processes and to obtain the publicly available code, visit http://powhegbox.mib.infn.it/

\section{Results for $t \bar{t}+$ jet}

In the following we concentrate on the recent implementation of the $t \bar{t}+$ jet hadroproduction presented in Ref. [10]. The Large Hadron Collider (LHC) and the Tevatron provide a unique experimental environment for top-quark physics. Moreover, a large fraction of the inclusive $t \bar{t}$ production does actually contain events with one or even more additional jets. Due to the larger phase space available, the relative importance of data samples with $t \bar{t}+$ jets is larger at the LHC with respect to the Tevatron, increasing the need of an accurate theoretical description of this process. Top-quark pair-production associated with jets is also an important background to Higgs boson production in vector boson fusion and for many signals of new physics. The implementation reported here is based on the NLO QCD corrections evaluated in Ref. [11, 12], merged with HERWIG [13] and PYTHIA [14] SMC programs, using the POWHEG BOX. 


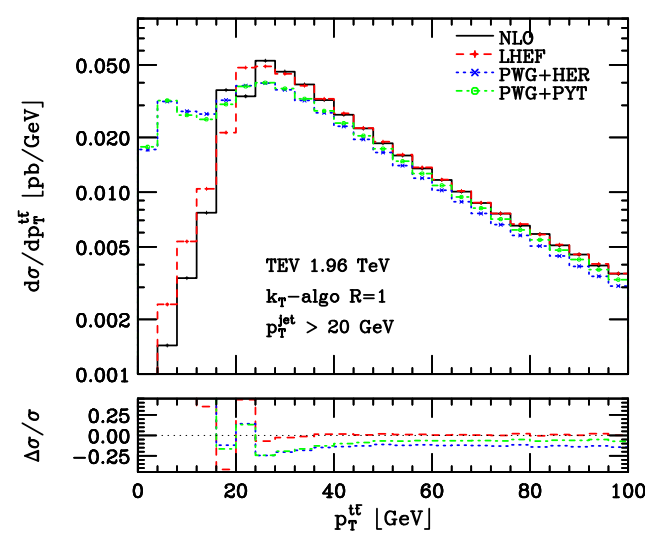

Figure 1: Differential cross section as a function of the $t \bar{t}$-pair transverse momentum at the Teva$\operatorname{tron}(\sqrt{s}=1.96 \mathrm{TeV})$

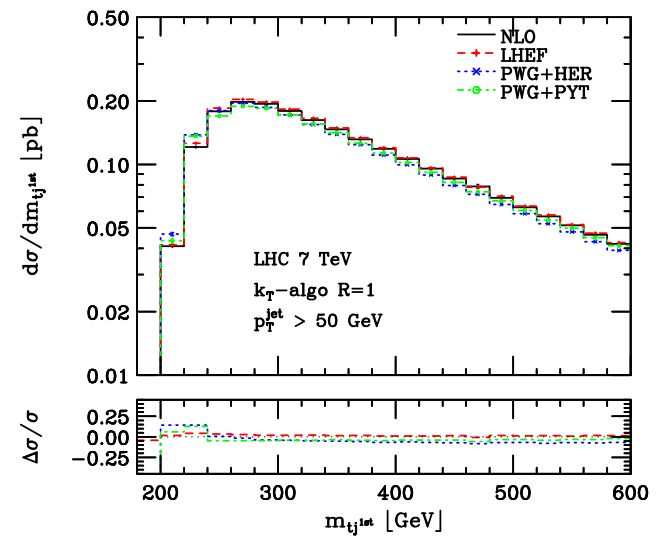

Figure 2: Differential cross section as a function of the $\left(t j_{1}\right)$ invariant mass at the $\operatorname{LHC}(\sqrt{s}=$ $7 \mathrm{TeV}$ )

We present results for both Tevatron and LHC colliders, having assumed a jet reconstruction cut in the analysis of $p_{\mathrm{T}}>20 \mathrm{GeV}$ and $50 \mathrm{GeV}$, respectively. We have used the inclusive- $k_{T}$ jet algorithm with $R=1$ and the $E_{T}$-recombination scheme. Renormalization and factorization scales have been set to $\mu_{R}=\mu_{F}=m_{t}=174 \mathrm{GeV}$, we have used the PDF set CTEQ6M, and we have not impose any extra acceptance cut, other than those necessary to define the hard jet.

In Fig. $\square$ we show the differential cross section as a function of the transverse momentum of the $t \bar{t}$-pair at the Tevatron, while in Fig. $\square$ we plot the invariant mass of the system made by the top-quark and the hardest jet at the $7 \mathrm{TeV}$ LHC. The different curves appearing on each plot refer respectively to the fixed order results (NLO), to the results after the first emission has been performed by POWHEG (LHEF) and to the fully showered events, with HERWIG (PWG+HER) Or PYTHIA (PWG+PYT) showers. Shower effects are visible in the low- $p_{\mathrm{T}}^{t \bar{t}}$ region, while more inclusive observables like the invariant mass of the system made by the top-quark and the hardest jet, $m_{\left(t j_{1}\right)}$, are basically unaffected by the shower.

In our implementation we have also included the spin-correlations between the production and decay stages. In doing so, we have neglected off-shell effects and non-resonant production mechanisms. We proceeded by first generating events with stable top-quarks (un-decayed events) through the usual POWHEG machinery and then generating the decay products according to the matrix element for the full production and decay process (decayed events), following Ref. [15]. In our study we always assumed the semi-leptonic top-quark decay channel $t \rightarrow W^{+} b \rightarrow \ell^{+} v b$. In Fig. B] we draw the differential distribution $\frac{1}{\sigma} \frac{d^{2} \sigma}{d \cos \theta_{1} d \cos \theta_{2}}$ after the HERWIG shower, at the Tevatron collider, with $\theta_{1}$ and $\theta_{2}$ being the angles between the directions of the flights of the leptons coming from the decayed top-quark in the $t(\bar{t})$ rest frame and the beam axis, whose direction defines the quantization axis for the (anti-)top-quark spin. No extra acceptance cut is imposed on the leptons. In Fig. $\theta$ we show instead the differential cross section as a function of the azimuthal distance between the two leptons coming from the top-quarks decays, for the LHC collider configuration and after the PYTHIA shower. An extra cut $m_{t \bar{t}}<400 \mathrm{GeV}$ has been imposed here to enhance the effect. Looking at both plots, it is possible to appreciate the differences between spin-correlated results and those obtained by letting the respective SMC program performing the uncorrelated top-quark decays. 


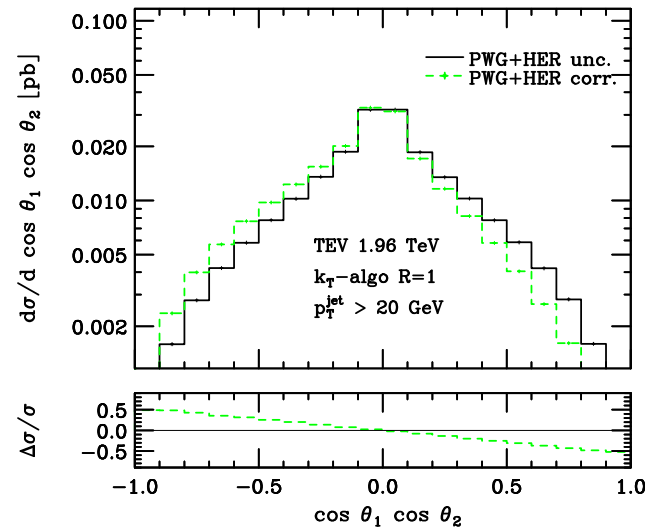

Figure 3: Effect of the inclusion of spin correlations when interfacing to HERWIG.

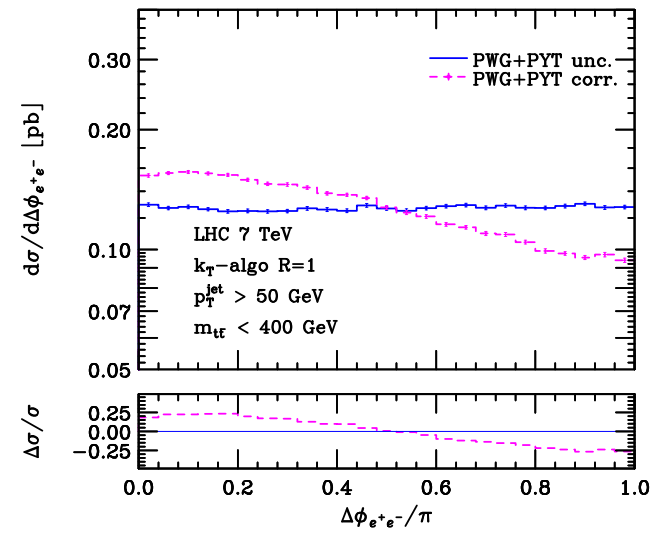

Figure 4: Effect of the inclusion of spin correlations when interfacing to PYTHIA.

We have also investigated the $t \bar{t}$ charge asymmetry in presence of a hard jet, finding that the inclusion of the shower changes significantly the fixed-order predictions in the low $p_{T}^{t \bar{t}}$ region, where shower effects are known to be large. Away from this region the parton shower leads only to a marginal change of the charge asymmetry binned in $p_{T}^{t \bar{t}}$. This quantity is now available at NLO accuracy, supplemented by the shower. For more details and for complete tables including results obtained with different cuts and at various stages of the simulation, we refer to Ref. [10].

\section{References}

[1] S. Frixione and B. R. Webber, JHEP 0206 (2002) 029 [arXiv:hep-ph/0204244].

[2] P. Nason, JHEP 0411 (2004) 040 [arXiv:hep-ph/0409146].

[3] S. Frixione, P. Nason and C. Oleari, JHEP 0711 (2007) 070 [arXiv:0709.2092 [hep-ph]].

[4] S. Alioli, P. Nason, C. Oleari and E. Re, JHEP 1006 (2010) 043 [arXiv:1002.2581 [hep-ph]].

[5] S. Alioli, K. Hamilton, P. Nason, C. Oleari, E. Re, JHEP 1104 (2011) 081. [arXiv:1012.3380 [hep-ph]].

[6] T. Melia, P. Nason, R. Rontsch, G. Zanderighi, Eur. Phys. J. C71 (2011) 1670. [arXiv:1102.4846 [hep-ph]]

[7] B. Jager, G. Zanderighi, [arXiv:1108.0864 [hep-ph]].

[8] T. Melia, P. Nason, R. Rontsch, G. Zanderighi, [arXiv:1107.5051 [hep-ph]].

[9] C. Oleari, L. Reina, JHEP 1108 (2011) 061. [arXiv:1105.4488 [hep-ph]].

[10] S. Alioli, S. -O. Moch, P. Uwer, [arXiv:1110.5251 [hep-ph]].

[11] S. Dittmaier, P. Uwer, S. Weinzierl, Phys. Rev. Lett. 98 (2007) 262002. [hep-ph/0703120 [HEP-PH]].

[12] S. Dittmaier, P. Uwer, S. Weinzierl, Eur. Phys. J. C59 (2009) 625-646. [arXiv:0810.0452 [hep-ph]].

[13] G. Corcella et al., JHEP 0101 (2001) 010 [arXiv:hep-ph/0011363].

[14] T. Sjostrand, S. Mrenna and P. Z. Skands, JHEP 0605 (2006) 026 [arXiv:hep-ph/0603175].

[15] S. Frixione, E. Laenen, P. Motylinski, B. R. Webber, JHEP 0704 (2007) 081. [hep-ph/0702198 [HEP-PH]]. 